\title{
MULTICOLOR QUANTUM DOT-BASED FLUORESCENCE EXCITATION FOR CELLULAR MICRO-ARRAY SCREENING

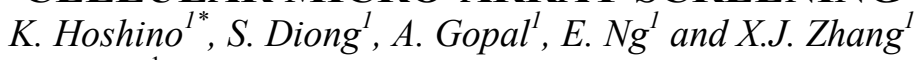 \\ ${ }^{1}$ Department of Biomedical Engineering, The University of Texas at Austin, Austin, Texas, USA
}

\begin{abstract}
We have developed a colloidal quantum dot (QD) - based multicolor excitation light source array designed for high throughput lab-on-a-chip cell screening and imaging. We have demonstrated preliminary measurements of fluorescence excitation that is suitable for the arrayed fluorescence immunoassay. Ex vivo transmission microscopy and fluorescence imaging of cultured cancer cells (MDA-MB 231 and SKBr3) were performed to demonstrate the efficacy of the QD-based light source. Our lowcost light source array is highly compatible with commercially available fluorescence microscopes and fluorescent markers commonly used for biomedical imaging and screening.
\end{abstract}

\section{INTRODUCTION}

Colloidal quantum dots (QDs) have demonstrated significant potential as fluorescent markers for bioimaging and sensing [1-3]. Emission wavelengths can be easily tailored by proper choice of materials and the size of the QDs. Semiconductor QDs are much more stable and slow to photobleaching compared with commonly used organic fluorescent dyes.

Here we demonstrate another important advantage of colloidal QDs, which is the compatibility with the advanced microfabrication technologies. In previous studies, we have shown patterning of colloidal QDs on silicon substrates [4-5]. The feature size of patterned QDs can be arbitrary chosen from millimeter scale down to single molecular order. Those patterned QDs can be also used as lumophores of light emitting diodes (LEDs) directly integrated onto silicon microdevices. We constructed QDLEDs onto several types of silicon MEMS structures [6].

One strong potential application for integrated QD light source is lab-on-a-chip bioimaging systems [6-7]. Hyperspectral analysis of cells using multiple fluorescent markers demonstrated efficacy in identification of cancer cell types [8]. In such immunofluorescence bioimaging systems, multiple excitation light source is crucial to efficiently excite different types of fluorescent markers that have different absorption wavelength bands. Colloidal QDs easily enable integration of multicolor sources on a single substrate. Furthermore, QD based illumination systems are highly compatible with solid state excitation configuration. colloidal QDs can be either electrically excited or excited by an UVLED. Solid state light sources have been drawing attention for imaging and spectroscopy [9-10], because it can construct a highly integrated, low-cost, energy efficient illumination setup. It also enables time controlled fluorescence measurements such as fluorescence lifetime measurement [9].

In this paper, we propose a colloidal QD based multicolor light source designed for fluorescence biosample imaging. Excited by a high power UVLED, patterned Colloidal QDs work as an excitation source for transmission microscopy and immunofluorescent cell imaging.

\section{EXPERIMENTAL SETUP}

Patterned QD light source

The two key techniques we introduced here are patterning of colloidal QDs on a glass substrate and UV excitation utilizing total internal reflection in the glass substrate. Figure 1 shows an illustration of the fabrication procedure and the experimental setup. Array of colloidal QD patterns were fabricated onto a glass slide by the micro contact printing technique. The colloidal QDs are $\mathrm{CdSe} / \mathrm{ZnS}$ core-shell nanoparticles. PDMS stamps formed by SU-8 patterned molds were used to transfer films of quantum dots onto the substrate. The details of the stamping techniques are described in [5], where we have previously demonstrated patterning of QDs as fine as $500 \mathrm{~nm}$. An UV light from a high-power LED (LedEngin, $200 \mathrm{~mW}$ ) with the emission wavelength peaked at $405 \mathrm{~nm}$ or 365 $\mathrm{nm}$ is focused and coupled to the substrate glass slide to induce total internal reflection. The QD films are excited by the UV evanescent field on the surface of the glass slide.

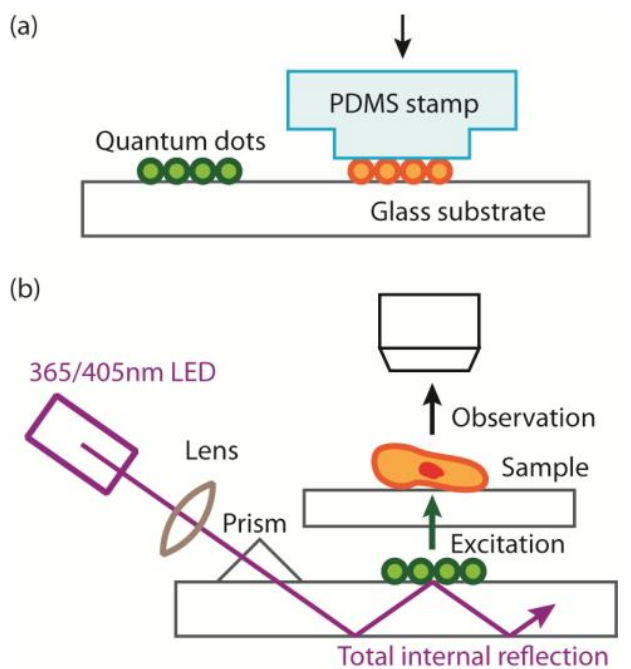

Figure 1: (a) Fabrication procedure and (b) experimental setup of the arrayed multicolor light source

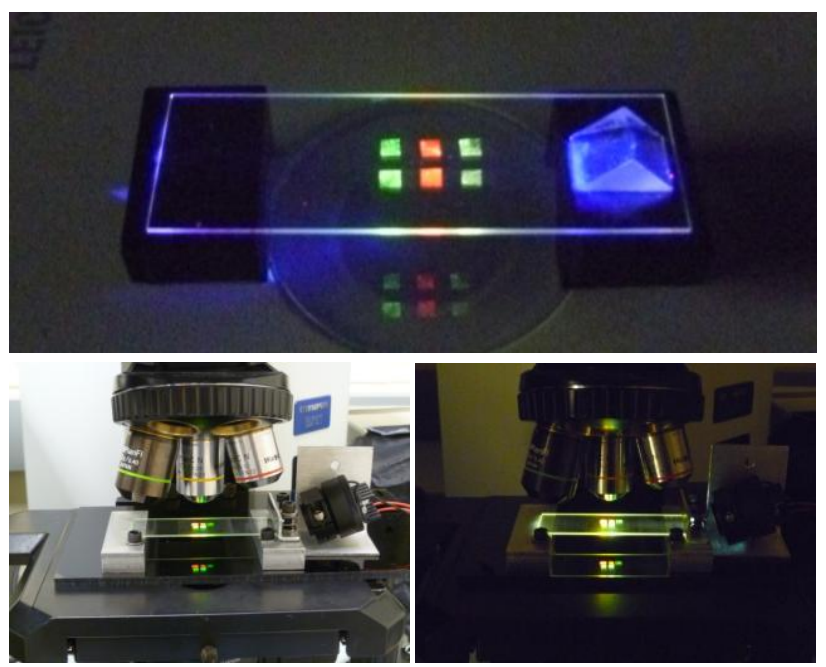

Figure 2: Emission from the $Q D$ array and experimental setup with a standard fluorescence microscope 
Figure 2 shows photographs of the emission from the QD arrays and the experimental setup built in a standard fluorescence microscope (Olympus BX51). Because the evanescent field decays quickly in the near-field and does not transmit energy in the farfield, intensities of the UV light observed with the microscope is almost negligible compared with those of the QDs that are excited by the evanescent field. Figure 3 shows the emission spectra of the QD array. Peak emission wavelengths can be easily chosen by using QDs with a proper average diameter.

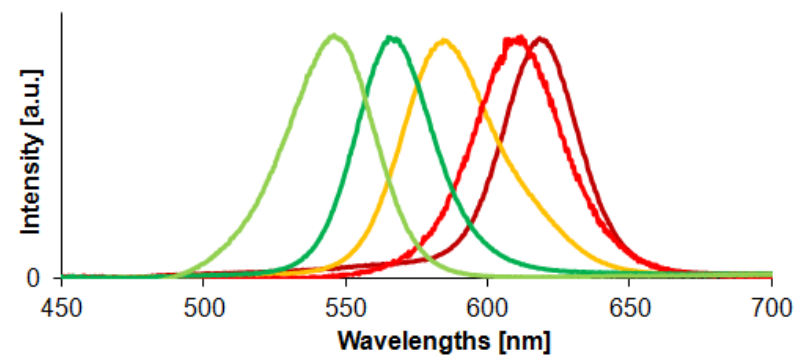

Figure 3. Emission spectra of the $Q D$ array. Emission peaks ranging from $540 \mathrm{~nm}$ to $620 \mathrm{~nm}$ were used in the measurement.

\section{Fluorescence excitation measurement}

Figure 4 shows the setup the fluorescence excitation experiment. QDs with an emission peak at $540 \mathrm{~nm}$ and $655 \mathrm{~nm}$ were used as the excitation source (light source QDs) and the fluorescent marker (sample QDs), respectively. The sample QD is located on a $1 \mathrm{~mm}$-thick glass slide, which roughly defines the distance between the excitation QD light source and the sample QD. A UV-cut plastic thin film and a green color film filter are inserted between the light source QD and the sample QDs. The purposes of these filters are: (1) To avoid cross direct excitation of sample QDs by stray lights from the UV LED and (2) To remove crosstalk from the light source QD to the fluorescence from the sample QD. A microscopic spectrometer consisting of a monochromator (Princeton Instruments Acton SP2150) and a cooled CCD (Princeton Instruments Pixis 400) measured the fluorescence from the sample QDs along with the excitation from the light source QDs.

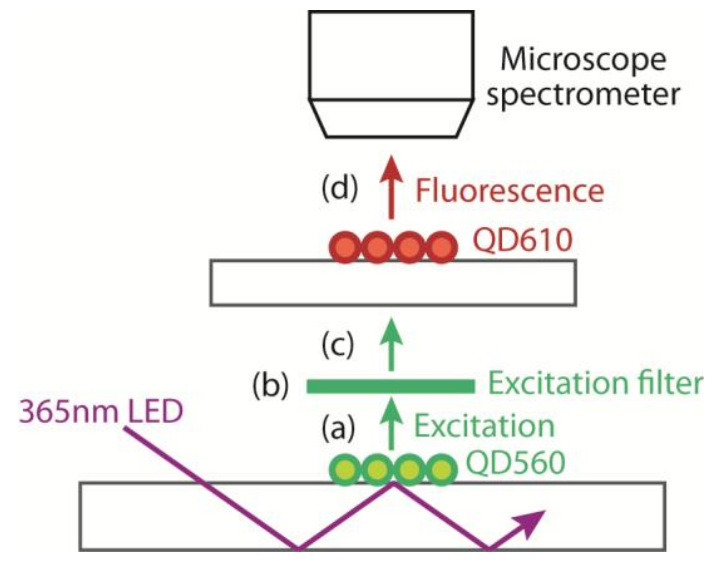

Figure 4: Fluorescence excitation setup with the light source $Q D$ (emission peaked at $560 \mathrm{~nm}$ ) and sample QD (emission peaked at $610 \mathrm{~nm})$.
Figure 5(a) shows the spectrum measured for the emission of the light source QDs (QD540) without any filter attached (see also figure 4(a) for the setup). One common issue of using two-step excitation as introduced here is cross excitation from the primary excitation source. As one can see in figure 2 top, some UV emission is visible at the edges of the slide glass and the prism. These stray lights may cause unwanted cross excitation of the sample QDs. In the actual experimental setup, a black shield box was attached to cover the observation area to block stray lights from the UVLED. In the area around the light source QD, scattering of UV light is small enough and not visible. Direct emission from UVLED cannot be found in the spectrum in figure 5(a). Another issue is the crosstalk between the light source QD and the fluorescence of the sample QD. In figure 5(a), detectable emission is found in the range of $>600 \mathrm{~nm}$, which will interfere with the observation of fluorescence from the sample. A green filter is used to trim the portion of $>600 \mathrm{~nm}$ emission. Transmission spectrum of the green filter is shown in the figure 5(b). Figure 5(c) is the spectrum of the light source QD with the filters attached. The half width of excitation in figure $5(\mathrm{~d})$ was $25 \mathrm{~nm}$, and is narrower than $29 \mathrm{~nm}$ measured for the emission from the light source QD. Figure 5(d) is the spectrum measured for the sample QD, along with the excitation light from the QD through the green and UVcut filters. Fluorescence from the sample QDs (QD620) is clearly visible in the spectrum.

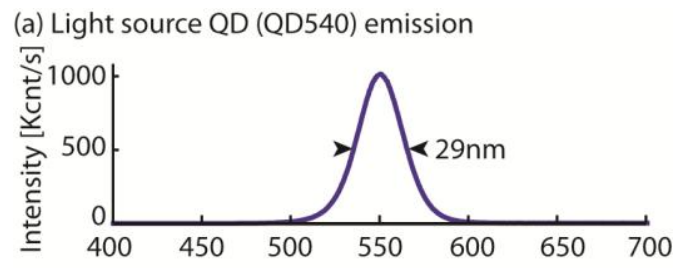

(b) Filter transmission
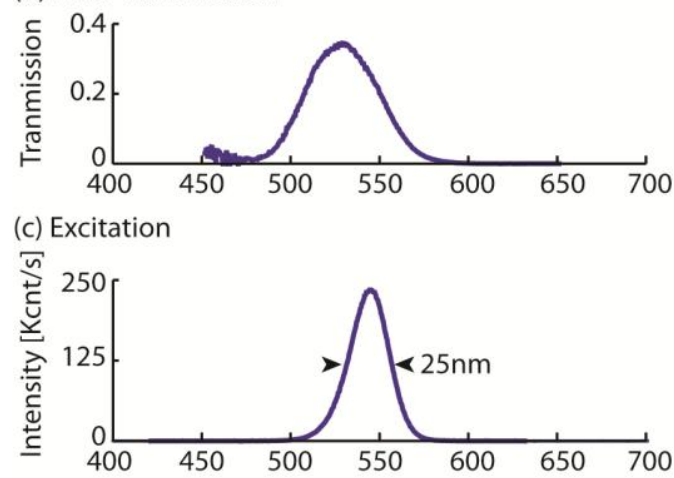

(d) Excitation and fluorescence

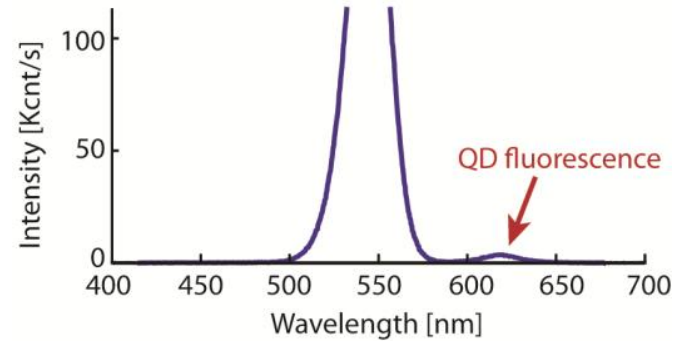

Figure 5: Spectrum showing the QD excitation (peaked at 540nm) and the fluorescence (peaked at610nm) 


\section{Power density of the QD light source}

The power density of the QD light source can be evaluated by the photon count measured by the spectrometer. The emission power $p_{i}$ of a single photon with a wavelength of $\lambda_{i}$ is:

$$
p_{i}=h v=h \cdot \frac{c}{\lambda_{i}}
$$

where $h$ is the Planck constant, $c$ is the speed of light. The emission energy measured by the CCD can be obtained by adding up energies measured for all the wavelengths of $\lambda_{i}(i=1 \sim N)$

$$
p=\sum_{i=1}^{N} p_{i} \cdot \text { count }_{i},
$$

Where count is the value of photon count per second for a wavelength $\lambda_{i}$.

The microscope objective collects only a part of the entire QD emission. We use an objective with a numerical aperture (NA) of $\sin \theta=0.65$. The emission $P_{\mathrm{m}}[\mathrm{W}]$ from the measured area is:

$$
P_{\mathrm{m}}=p \cdot(4 \pi) / 2 \pi \int_{0}^{\theta} \sin \varphi \cdot \mathrm{d} \varphi .
$$

The microscope spectrometer measures the intensity of lights through an opening slit that corresponds to $15 \mu \mathrm{m} \times 170 \mu \mathrm{m}$ on the sample plane. The power density $\left[\mathrm{W} / \mathrm{cm}^{2}\right]$ is thus given as

$$
P=P_{\mathrm{m}} \cdot 10^{-2} \times 10^{-2} /\left(15 \cdot 10^{-6} \times 170 \cdot 10^{-6}\right)
$$

Applying (1)-(4) to the measurement in figure 5(c), the power density of the QD at a point (c) becomes:

$$
P=2.1 \times 10^{-6}\left[\mathrm{~W} / \mathrm{cm}^{2}\right]
$$

\section{CANCER CELL IMAGING}

\section{Transmission-mode illumination}

The QD light source was first used as a transmission-mode illumination source to perform observation of cancer cells. A $150 \mathrm{~m}$-thick cover glass slip, on which cancer cells are fixed, is placed on a QD light source. The cells are stained with HEMA 3 (Fisher Scientific), which is commonly used to visualize cell nucleus. HEMA 3 contains eosin Y that stains the cytoplasm and methylene blue that stains the nucleus of the cell. The dye absorbs visible light and makes the stained area visible under transmissionmode illumination. The transmission spectrum of the HEMA3 dye is shown in figure 6 .

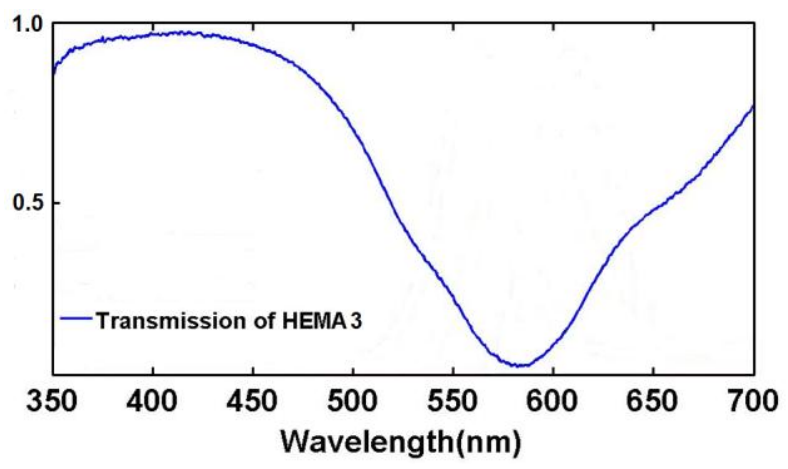

Figure 6: Absorption spectrum of the HEMA3 dye. It absorbs lights in visible range of roughly 540-620nm.

We can use a light source with emission wavelengths complementarily peaked in the absorption peak of HEMA 3 dye to clearly observe the stained area. Absorption peak of HEMA3 ranges roughly in the visible region of 540-620nm. Figure 7 shows an example of cells imaged with the multicolor light sources. MDA-MB231 breast cancer cells were used as the sample. We were able to clearly observe and determine the nucleus to cytoplasm ratio, which is an important factor to distinguish cancer cells from normal cells.
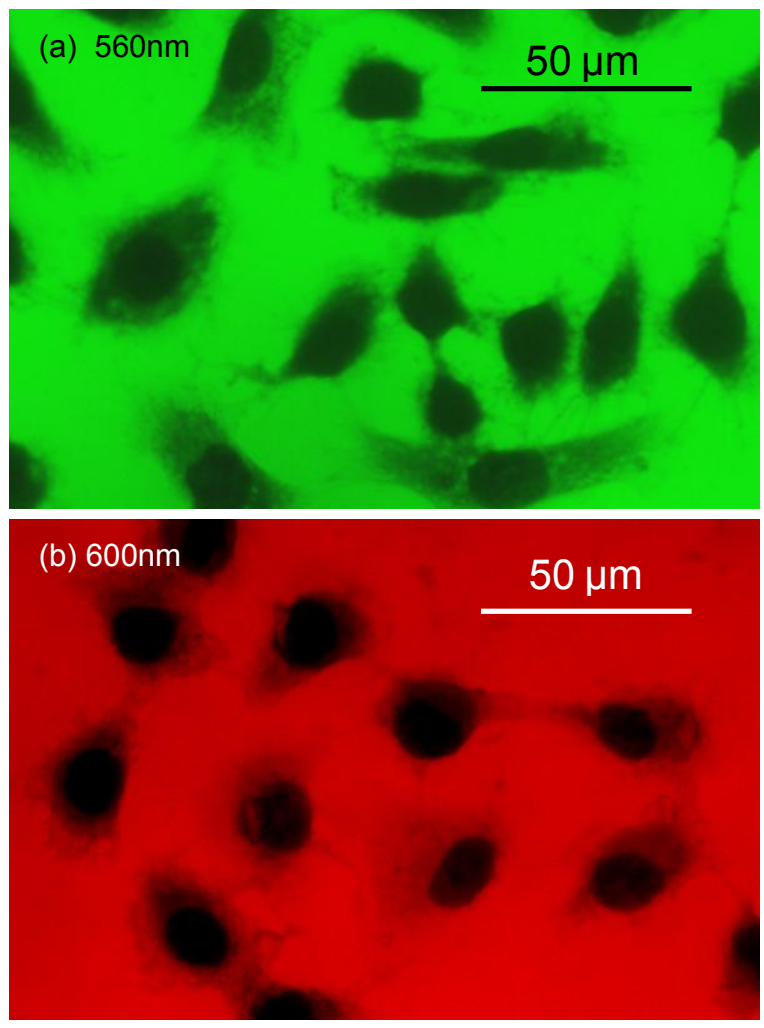

Figure 7: MDA-MB232 cells imaged with transmission mode illumination with (a) QD560 and (b) QD600. The cells are stained with the HEMA3 dye.

\section{Fluorescence excitation}

We performed fluorescence imaging of cultured cancer cells. A breast cancer cell line $\mathrm{SKBr} 3$ was used. SKBr3 is known to overexpress the gene product of Human Epidermal Growth Factor Receptor 2 (HER2).
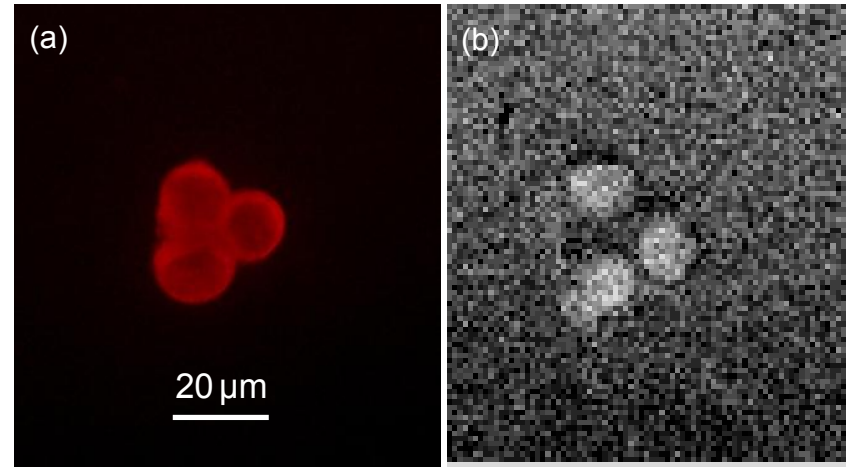

Figure 8. Fluorescence images of SKBr3 cancer cells. (a) Imaged with a standard fluorescence excitation setup. (b) Imaged with QD540 excitation source. 
Her2 is often used as a biomarker for cancer cell identification or targeted therapy, since Her2 overexpression is found in a certain aggressive type of breast cancer. Her2 is thus clinically used to characterize the type of breast cancer. Figure 8 (a) is a fluorescence image of $\mathrm{SKBr} 3$ cells taken with a standard fluorescence microscope (Olympus BX51). After the cultured cells are trypsinized from a culture dish, they were fixed on a glass slide and stained with AlexaFluor 594 dye conjugated with Her2 antibodies. A color CCD camera (Olympus DP71) and a filter cube (excitation peak wavelength $535 \mathrm{~nm}$, bandwidth $50 \mathrm{~nm}$, dichroic mirror $565 \mathrm{~nm}$ long-pass and emission peak wavelength $610 \mathrm{~nm}$, bandwidth $75 \mathrm{~nm}$ ) were used for Figure $8(\mathrm{a})$. Figure 8 (b) is a fluorescence image taken with the QD540 excitation light source. The same filter cube as figure 8(a) was used for fluorescence observation. A cooled monochromatic CCD (Princeton Instruments Pixis 400) was used for imaging. Although the image is dimmer compared with the standard excitation setup, the same cells are fluorescently observed with our integrated QD light source.

\section{CONCLUSION}

We have developed an arrayed colloidal QD excitation light source that can be easily used in commercially available microscopic observation setup. We characterized the QD light source by fluorescence excitation measurements of test QD samples. The setup is then successfully used for imaging of ex-vivo cancer cell transmission-mode illumination microscopy and immunofluorescence imaging.

Our arrayed excitation is especially suitable for patterned immuno-fluorescent assay [11-12] or cellular microarray imaging [13], where multiple biomarkers are tested in a microarrayed configuration. The QDs used here can be electrically excited in an LED configuration, which will further extend the possibility and versatility of our illumination setup.

\section{REFERENCES}

[1] X. Wu, H. Liu, J. Liu, K.N. Haley, J.A. Treadway, J.P. Larson, N. Ge, F. Peale, and M.P. Bruchez, "Immunofluorescent labeling of cancer marker Her2 and other cellular targets with semiconductor quantum dots," Nature Biotechnology 21, 41-46, 2003.

[2] X. Michalet, F. F. Pinaud, L.A. Bentolila, J.M. Tsay, S. Doose, J.J. Li, G. Sundaresan, A.M. Wu, S. S. Gambhir, S. Weiss, "Quantum Dots for Live Cells, in Vivo Imaging, and Diagnostics," Science 307, 538-544, 2005.

[3] I.L. Medintz, H.T. Uyeda, E.R. Goldman and H. Mattoussi, "Quantum dot bioconjugates for imaging, labelling and sensing," Nature Materials 4, 435-446, 2005.

[4] A. Gopal, K. Hoshino \& X.J. Zhang, "Photolithographic patterning of sub-wavelength colloidal quantum dot based inorganic light emitting diodes on silicon," Applied Physics Letters, 96, 13, 131109, 2010.

[5] K. Hoshino, T.C. Turner, S. Kim, A. Gopal and X.J. Zhang, "Single molecular stamping of self-assembled sub-10nm colloidal quantum dots," Langmuir 2008, 24, 13804-13808.

[6] A. Gopal, Z. Wang, K. Hoshino and X.J. Zhang, "Multispectral Analysis of Cancer Cells using Quantum Dot LEDs patterned on chip", The 16th International Conference on Solid-State Sensors, Actuators and Microsystems (Transducers 2011), pp.1817-1820.

[7] B. Yao, G. Luo, L. Wang, Y. Gao, G. Lei, K. Ren, L. Chen, Y. Wang, Y. Hub and Y. Qiu, "A microfluidic device using a green organic light emitting diode as an integrated excitation source," Lab on a Chip 5, 1041-1047, 2005.

[8] J.W. Uhr, M.L. Huebschman, E.P. Frenkel, N.L. Lane, R. Ashfaq, Huaying Liu, D.R. Rana, L. Cheng, A.T. Lin, G.A. Hughes, X.J. Zhang, H.R. Garner, "Molecular profiling of individual tumor cells by hyperspectral microscopic imaging," Translational Research 2011 (in press).

[9] T. Araki, H. Misawa, "Light emitting diodebased nanosecond ultraviolet light source for fluorescence lifetime measurements," Review of Scientific Instruments 66, 5469, 1995.

[10] E. Entcheva, Y. Kostov, E. Tchernev, L. Tung, "Fluorescence imaging of electrical activity in cardiac cells using an allsolid-state system," 51, $333-341,2004$

[11] E. Ng, A. Gopal, K. Hoshino, \& X.J. Zhang, "Multicolor Microcontact Printing of Proteins on Nanoporous Surface for Patterned Immunoassay," Applied Nanoscience, 1, 79-85, 2011.

[12] E. Blinka, K. Loeffler, Y. Hu, A. Gopal, K. Hoshino, K. Lin, X. Liu, M. Ferrari \& X.J. Zhang, "Enhanced Microcontact Printing of Proteins on Nanoporous Silica Surface," IOP Nanotechnology, 21, 415302, 2010.

[13] H.C. Moeller, M.K. Mian, S. Shrivastava, B.G. Chung \& A. Khademhosseini, "A microwell array system for stem cell culture," Biomaterials 29, 752-763, 2008.

\section{CONTACT}

*K. Hoshino, tel: +1-512-475-6872; hoshino@mail.utexas.edu 